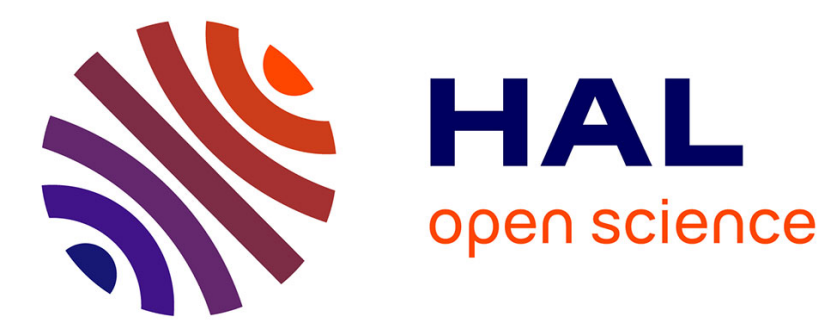

\title{
Phénomènes de transport atomique dans les alliages liquides indium-plomb
}

\author{
J.F. Rialland, J. Robert
}

\section{To cite this version:}

J.F. Rialland, J. Robert. Phénomènes de transport atomique dans les alliages liquides indium-plomb. Journal de Physique Lettres, 1975, 36 (4), pp.89-91. 10.1051/jphyslet:0197500360408900 . jpa00231163

\section{HAL Id: jpa-00231163 https://hal.science/jpa-00231163}

Submitted on 1 Jan 1975

HAL is a multi-disciplinary open access archive for the deposit and dissemination of scientific research documents, whether they are published or not. The documents may come from teaching and research institutions in France or abroad, or from public or private research centers.
L'archive ouverte pluridisciplinaire HAL, est destinée au dépôt et à la diffusion de documents scientifiques de niveau recherche, publiés ou non, émanant des établissements d'enseignement et de recherche français ou étrangers, des laboratoires publics ou privés. 


\title{
PHÉNOMẼNES DE TRANSPORT ATOMIQUE DANS LES ALLIAGES LIQUIDES INDIUM-PLOMB
}

\author{
J. F. RIALLAND et J. ROBERT \\ Laboratoire de Génie Electrique de Paris (L.G.E.P., L.C.I.E.), \\ 33, avenue du Général-Leclerc, 92260 Fontenay-aux-Roses, France
}

(Reçu le 28 octobre 1974, accepté le 13 février 1975)

\begin{abstract}
Résumé. - Les phénomènes de transport atomique sont interprétés dans le système indiumplomb à l'aide d'un modèle de sphères dures. A partir des données expérimentales, on obtient des diamètres de cœur dur de 3,04 $\AA$ pour l'indium et 3,40 Å pour le plomb. Les distances entre l'origine et le premier minimum des courbes représentatives des potentiels de paires sont respectivement de $3,1 \AA$ et $3,3 \AA$ pour l'indium et le plomb. Compte tenu de la précision de nos résultats, les diamètres de cœur dur obtenus sont compatibles avec ces valeurs.
\end{abstract}

\begin{abstract}
A hard sphere model is used to interpret atomic transport processes in indium-lead alloys. Our experimental data lead to hard core diameters of $3.04 \AA$ for indium and $3.40 \AA$ for lead. The distances between the origin and the first minimum of the pair potential curves are $3.1 \AA$ and 3.3. $\AA$ for indium and lead respectively. Thus, within the accuracy of the data, the results obtained from theory and experiment are in good agreement.
\end{abstract}

Dans les alliages binaires, le coefficient d'interdiffusion $D$ est relié aux coefficients d'hétérodiffusion $D_{\mathrm{i}}$ de chaque constituant par la relation de Darken [1] :

$$
D=\left(X_{2} D_{1}+X_{1} D_{2}\right) \frac{\mathrm{d} \log a_{1}}{\mathrm{~d} \log X_{1}}
$$

où $X_{1}$ et $X_{2}$ sont les fractions atomiques de chaque constituant et $a_{1}$ l'activité thermodynamique de l'espèce 1. Pour établir cette équation, il est nécessaire de supposer que les volumes molaires partiels ne dépendent pas de la concentration.

Les coefficients d'hétérodiffusion peuvent être exprimés à partir de la viscosité dynamique $\eta$ de l'alliage par la relation [2] :

$$
D_{\mathrm{i}}=\frac{k T}{\sigma_{\mathrm{i}} \eta} \quad i=1,2
$$

où $\sigma_{1}$ et $\sigma_{2}$ sont les facteurs de friction.

Dans la théorie hydrodynamique, on suppose que les atomes, sphériques, diffusent dans un milieu continu. A partir de la loi de Stokes, Lamb calcule $\sigma_{\mathrm{i}}$ pour les deux valeurs limites du coefficient de friction par glissement $\beta$ [3]. Les coefficients d'hétérodiffusion s'écrivent alors :

$$
\begin{aligned}
& D_{\mathrm{i}}=\frac{k T}{6 \pi r_{\mathrm{i}} \eta}(\beta=\infty) \\
& D_{\mathrm{i}}=\frac{k T}{4 \pi r_{\mathrm{i}} \eta}(\beta=0)
\end{aligned}
$$

où $r_{\mathrm{i}}$ est le rayon des sphères qui diffusent. L'éq. (3) est connue sous le nom de relation de Stokes-Einstein. Dans le cas des métaux liquides, il semble que la relation (4) est mieux adaptée [2]. L'éq. (1) s'écrit dans ces conditions :

$$
\frac{D \eta}{\alpha_{1} T}=\frac{k}{4 \pi}\left(\frac{X_{2}}{r_{1}}+\frac{X_{1}}{r_{2}}\right)=\frac{k}{4 \pi}\left[\frac{1}{r_{1}}+X_{1}\left(\frac{1}{r_{2}}-\frac{1}{r_{1}}\right)\right]
$$

avec

$$
\begin{aligned}
& \alpha_{1}=\frac{\mathrm{d} \log a_{1}}{\mathrm{~d} \log X_{1}} \\
& X_{1}+X_{2}=1 .
\end{aligned}
$$

1. Application au système indium-plomb. - Les coefficients d'interdiffusion sont déterminés par la méthode de Boltzmann-Matano [4], à partir des profils de diffusion [5].

Lorsque les volumes molaires partiels de chaque constituant ne sont pas égaux, les concentrations doivent être exprimées dans l'équation de Matano en nombre d'atomes par unité de volume. Ceci implique une conversion des fractions atomiques en concentrations molaires. Si les volumes molaires partiels sont indépendants de la concentration, on obtient alors un coefficient d'interdiffusion $D$ unique.

Il est possible d'exploiter directement les profils de diffusion exprimés en fraction atomique. L'application de l'équation de Matano conduit alors à deux 
coefficients $D^{(1)}$ ou $D^{(2)}$ selon que les concentrations sont exprimées par rapport à l'espèce 1 ou 2. Lorsque les volumes molaires partiels sont constants, le calcul permet de déduire le coefficient d'interdiffusion $D$ de $D^{(1)}$ ou $D^{(2)}$ par l'équation [6] :

$$
D=\frac{v}{v_{1}} D^{(2)}=\frac{v}{v_{2}} D^{(1)}
$$

où $v$ est le volume molaire de l'alliage, $v_{1}$ et $v_{2}$ les volumes molaires partiels.

Les volumes molaires partiels de l'indium et du plomb étant différents, nous avons appliqué à nos résultats cette dernière méthode. Les coefficients $D^{(\mathbf{P b})}$ sont tout d'abord déterminés à partir des profils de diffusion exprimés en fraction atomique en plomb [5]. Les coefficients d'interdiffusion sont alors obtenus à l'aide de la relation (6). Les volumes molaires sont calculés à partir des valeurs de la densité $[7,8]$. On vérifie que la loi de variation du volume molaire de l'alliage avec la concentration atomique est linéaire et, en conséquence, que les volumes molaires partiels de l'indium et du plomb ne dépendent pas de la concentration. Ce résultat entraîne l'unicité de $D$, donc la validité de la méthode utilisée. Sur la figure 1 sont portés les coefficients d'interdiffusion en fonction de la fraction atomique en plomb pour différentes températures. La précision sur les valeurs de $D$ est de. $5 \%$.

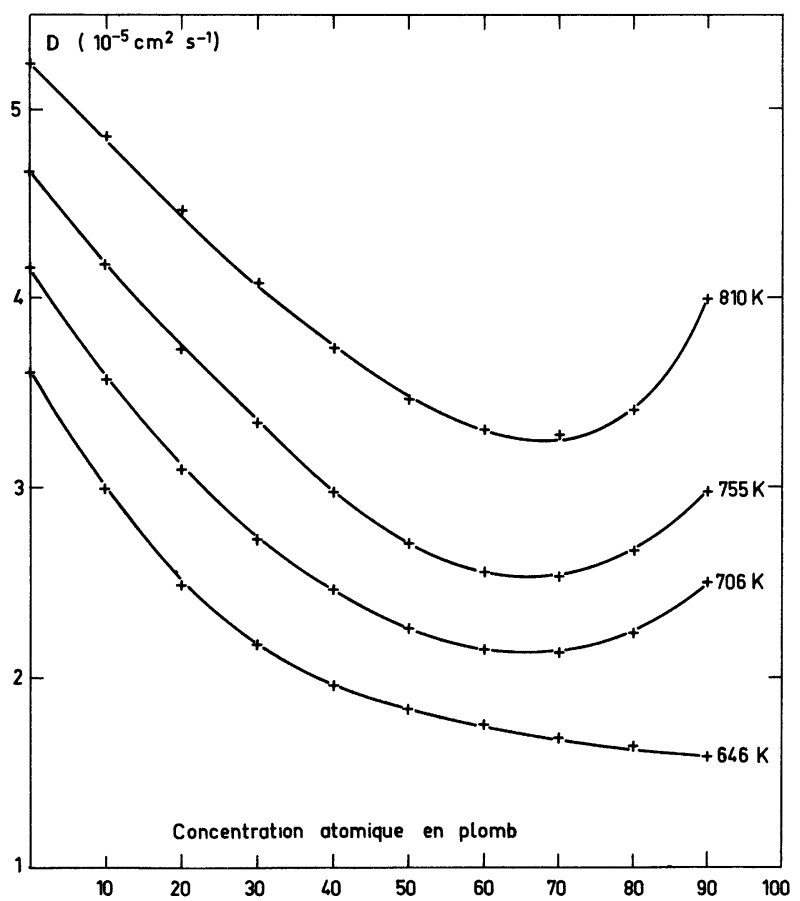

FIG. 1. - Variation du coefficient d'interdiffusion en fonction de la concentration atomique en plomb.

A partir de ces résultats et des valeurs de la viscosité [9], et de l'activité [10,11], nous avons calculé l'expression

$$
\frac{D \eta}{\alpha_{\mathrm{Pb}}}=f\left(X_{\mathrm{Pb}}, T\right)
$$

La précision sur les valeurs calculées peut être évaluée à $15 \%$. Dans le domaine de température étudié et compte tenu de la précision obtenue, la loi de variation de l'expression (7) avec la température absolue peut être considérée comme linéaire. (A titre d'exemple, nous avons porté sur la figure 2 la variation de l'expression (7) avec la température absolue dans le cas d'un alliage dont la concentration atomique en plomb est de 0,5). Ceci est en accord avec le modèle présenté. En effet, les rayons de sphères dures varient peu avec la température et le rapport

$$
\frac{D \eta}{T \alpha_{\mathrm{Pb}}}
$$

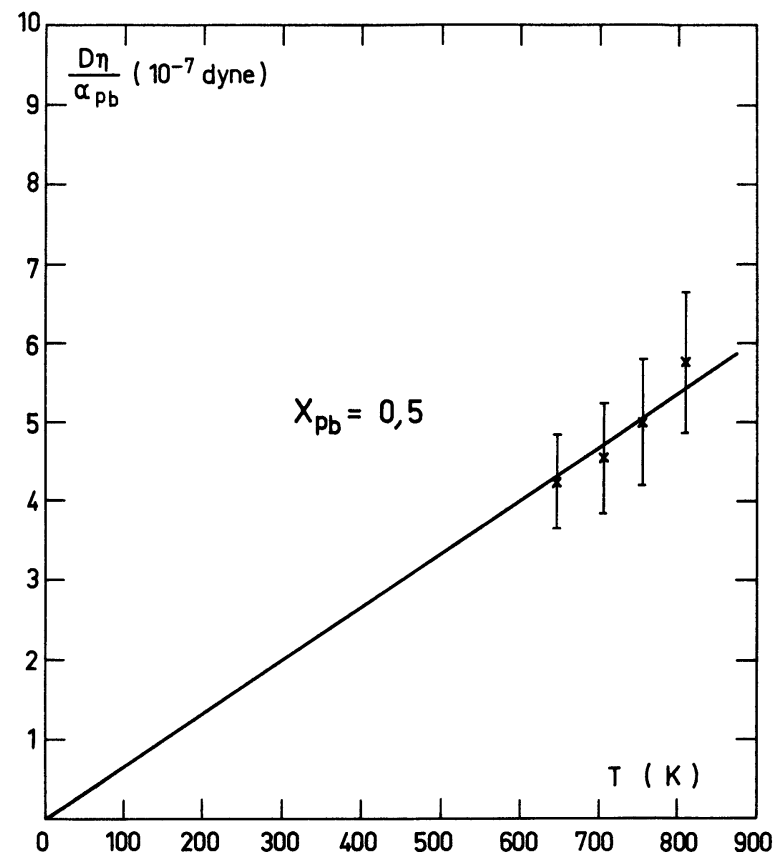

Fig. 2. - Variation de $D \eta / \alpha_{\mathrm{Pb}}$ en fonction de la température absolue.

doit apparaître, en première approximation, indépendant de $T$. Sur la figure 3 sont portées les variations de (8) en fonction de la fraction atomique en plomb. Sur la même figure, nous avons tracé la droite pour laquelle la somme du carré des écarts avec les points expérimentaux est minimum. La droite obtenue donne une bonne approximation des variations expérimentales. L'ordonnée à l'origine et la pente de cette droite permettent d'obtenir à partir de l'éq. (5) les rayons de sphères dures de l'indium et du plomb :

$$
r_{\mathrm{In}}=1,52 \AA \quad r_{\mathrm{Pb}}=1,70 \AA .
$$

Les fractions atomiques en indium et en plomb ayant un rôle symétrique dans les équations, l'exploitation des résultats par rapport à la concentration en indium conduirait aux mêmes rayons de sphères dures. Les distances entre l'origine et le premier minimum des courbes représentatives des potentiels de paires calculées à partir des fonctions de distribution radiale sont dans le cas de l'indium et du plomb [12] :

$$
d_{\mathrm{In}}=3,1 \AA \quad d_{\mathrm{Pb}}=3,3 \AA .
$$




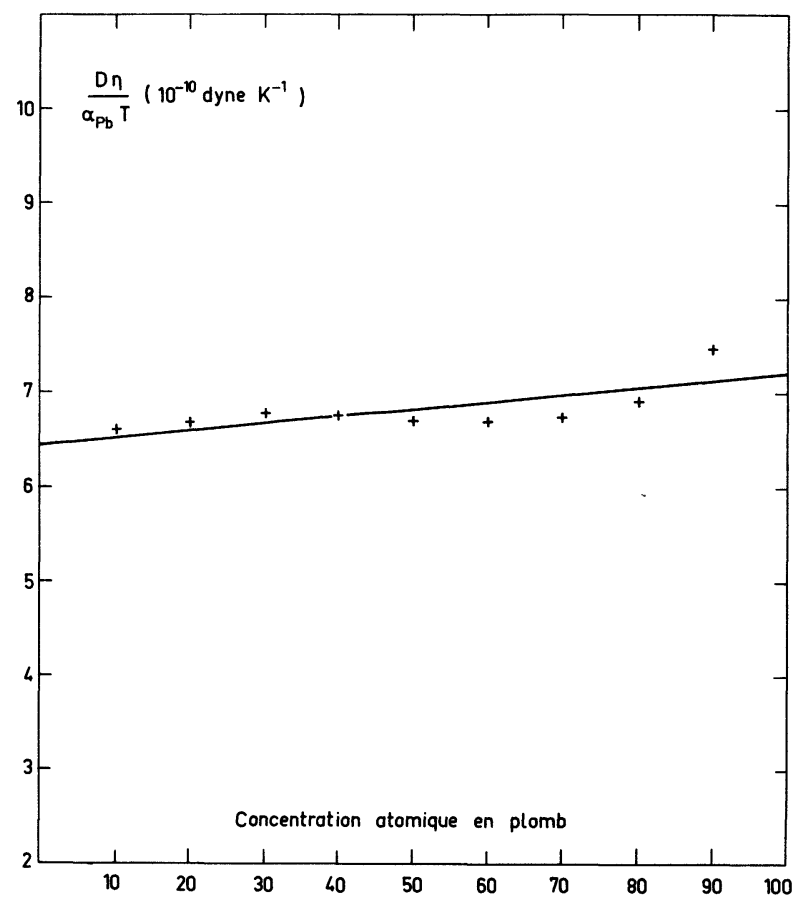

Compte tenu de la précision de nos résultats, les diamètres de sphères dures calculées à l'aide de l'éq. (5) sont compatibles avec les valeurs obtenues à partir des fonctions de distribution radiale. Ce résultat semble confirmer la validité de l'éq. (4) pour décrire les coefficients d'hétérodiffusion.

$\mathrm{Ce}$ modèle très simple semble traduire avec une bonne approximation les phénomènes de transport atomique dans le système indium-plomb. Néanmoins, il faut être prudent quant aux conclusions sur la validité de ce modèle. En effet, on attribue aux atomes une dimension géométrique qui ne dépend pas du milieu dans lequel ils se trouvent. En fait, il est évident que les interactions entre atomes interviennent. Ceci doit se traduire par la définition de rayons de sphères dures différents selon l'environnement considéré. Dans le cas du système étudié, la validité du modèle est sans doute due à l'absence de singularités notamment structurales.

Fig. 3. - Variation de $D \eta / \alpha_{\mathrm{pb}} T$ en fonction de la concentration atomique en plomb.

\section{Bibliographie}

[1] Darken, L. S., Trans.-AIME 175 (1948) 184.

[2] Ghai, R. K., Ertl, H., Dullien, F. A. L., Am. Inst. Chem. Eng. J. 19 (1973) 5, 881.

[3] Lamb, H., Hydrodynamics (Dover Publications) 1932.

[4] Crank, J., The Mathematics of diffusion (Oxford University Press) 1970.

[5] Rialland, J. F., Robert, J., Proc. 2d Int. Conf. Properties of Liquids Metals (Taylor and Francis, London) 1973.
[6] Potard, C., Tellier, A., Dusserre, P., Mat. Res. Bull. 7 (1972) 583.

[7] Predel, B., Emam, A., J. Less-Common Met. 18 (1969) 385.

[8] Berthou, P. E., Tougas, R., Met. Trans. 1 (1970) 2978.

[9] Rialland, J. F., Perron, J. C., Met. Trans. 5 (1974) 2401.

[10] TerpilowsKi, J., GregorCZyK, Z., Arch. Hutnictwa 6 (1961) 1977.

[11] Shiu, D. H., Munir, Z. A., High Temp. Sci. 3 (1971) 381.

[12] WasedA, Y., Suzuki, K., Phys. Stat. Sol. (b) 57 (1973) 351. 